\title{
The Scanning Photogrammetry
}

\author{
Tao Ke ${ }^{\mathrm{a}}$, ZuXun Zhang ${ }^{\mathrm{a}}$, Shan Huang ${ }^{\mathrm{a}, *}$ \\ School of Remote Sensing and Information Engineering, Wuhan University, Wuhan 430079, China- \\ ketao@whu.edu.cn, zhangzx@cae.cn,yunshan_2008@126.com
}

Commission V/5, WG V/5

KEY WORDS: Scanning photogrammetry, Close-range Photogrammetry, Multi-baseline, Aerial triangulation, Engineering survey, Deformation monitoring.

\begin{abstract}
:
The paper proposes a new photogrammetry method, the scanning photogrammetry, to solve the problem that large targets can hardly be processed as a whole one in close-range photogrammetry. The method enlarges the view angle and intersection angle effectively by rotating camera in horizontal and vertical direction when photographing large targets. Meanwhile, it is a kind of multibaseline photogrammetry which increases matching reliability and improves the quality and quantity of observations. Besides, in order to acquire images automatically, we develop the photograph scanner which ensures the efficiency and quality of photography. And the scanning photogrammetry system has been successfully used in deformation monitoring of Wumen Circumvallation in the Forbidden City. In the experiments, data is processed automatically by classical triangulation and self-calibration bundle adjustment. The result proves that the precision can meet with the deformation monitoring requirements and data processing efficiency accomplishes to engineering measurement applications.
\end{abstract}

\section{INTRODUCTION}

Non-metric camera's resolution can meet with the close-range measurement tasks, thus it's possible to gain high measurement results with non-metric cameras (Chandler, 1999; Fraser et al., 1995; Peipe and Schneider, 1995). Moreover as the non-metric camera has the advantages of changeable focus lens, various photograph methods and low cost, making it widely used in close-range photogrammetry(Feng, 1982). The progress helps the inexperienced users to make effective use of digital photogrammetry(Jiang and Jauregui, 2010; Luhmann, 2010). With regard to the shortcomings of unknown interior elements and instable distortion, most researchers use self-calibration bundle adjustment to solve.

The simplest way of using automated digital photogrammetry is to design the photography so that 'normal' vertical coverage is obtained, only at very large scale. If the camera can be positioned vertically above the object of interest, then the normal processing sequence can be adopted and DEMs generated automatically (Chandler, 1999). But, no matter metric camera or non-metric camera, the small picture format leads to the view of the camera is small. This prevents the camera being emplaced in the traditional and preferred normal attitude, and only oblique photographs can be obtained. As normal photography makes the intersection angle small which lowers the intersect precision, the convergent photography increases intersection angle to get high precision. So the convergent photography is common in close-range photogrammetry. However, the change of parallax is volatile at a large range, and occlusions are common in close-range imagery. When the intersection angle is larger than 15 degree, it's difficult for automatic image matching(Zhang et al., 2007). Nowadays, many photogrammetry systems apply multi-baseline convergent photography to solve the contradiction that large intersection angle makes image matching difficult due to large distortion of stereo images while small intersection angle results in low intersection precision.

But multi-baseline convergent photography also exist other problems, especially is limited for large engineering object measurement. On the one hand, the photography scope of convergent photography at one time is limited, which limits its application. Though, the idea of dividing the target into different blocks to photograph is used to solve this problem, it leads to another problem that an amount of field operation has to be done to measure the additional ground control points for bridging the blocks. It will take more time and reduce the data processing efficiency. On the other hand, lots of workers gain images at will in close-range photography, which makes data hard to organize. Meanwhile, as the complex of the scene and mistaking operation when controlling photography angle by hand are easily occurred, it can lead to photographic gap or bad intersection angle. These are disadvantage to automatically match and get high precision, also go against the standardization measurement.

To the problems submitted above, we propose the scanning photogrammetry method and develop a corresponding data acquisition and processing system. The scanning photogrammetry advances panning and multi-baseline photography presented by professor Zhang in 2007(Zhang et al., 2007; Ke et al., 2007; Ke, 2008; Zhang, 2007). Panning and multi-baseline photography is to rotate camera in horizontal direction so that it only enlarges view angle of station in horizontal direction, which is not adaptable to high engineering object. Therefore, the scanning photography presents to rotate camera in both horizontal and vertical direction by scanner while acquiring images automatically. And we classify stations as base stations (long baseline) and assistant stations (short baseline) to implement multi-view photography. After the data is obtained, the aero-triangulation and self-calibration bundle adjustment are used for automatic data processing. In all, the scanning photogrammetry overcomes the shortcoming that the panning and multi-baseline photogrammetry only measures the low targets, and can measure the large objects and meet the requirements in deformation monitoring. 


\section{METHOD}

\subsection{The scanning photography}

We present the scanning photography to solve the non-metric camera's small view angle limits the area of object in an image. It enlarges the view angle of the station by rotating camera in both horizontal and vertical direction to gain image matrix. This increases one station's angle of view to ensure intersection precision as the largest intersection angle of one point is large enough.

In figure1, we can see, the scanning photography is like the digital aerial camera DMC that splices 4 simultaneously panchromatic images together to get a large format image. The differences between them are that the former gets images by one camera rotating and processes images as individual while the latter gets images by several fixed cameras simultaneously and it needs to splice images before data processing. As the camera rotation that each image on the same station has different elements of exterior orientation, the image overlap should be more than $20 \%$ which is much larger than $1 \%$ in DMC images. Meanwhile, the large overlap increases the redundant observations, is benefit to improve precision.

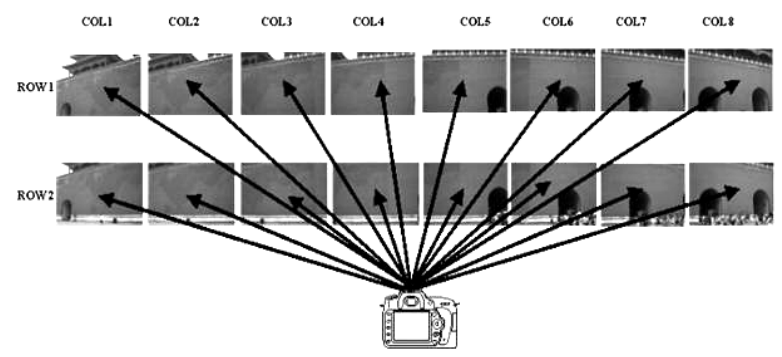

(a)

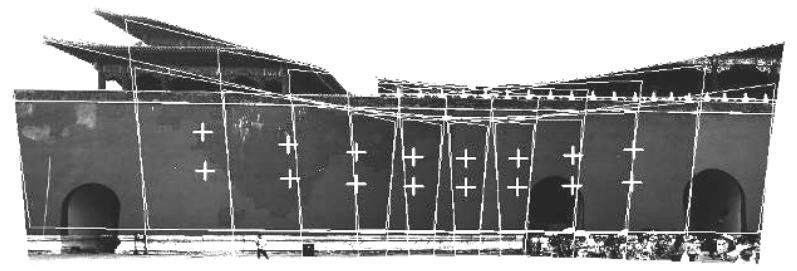

(b)

Figure1. Figure (a) shows the image matrix of 2 rows and 8 columns gained by scanning photography on one station. Figure (b) shows the mosaic Ortho-image from images in figure (a). And the white crossings present the positions of images' centre, the white lines shows the boundaries of each image.

As photographing, we need to make sure the photographic area of the station, calculate the quantity of rows and columns of image matrix according to the overlap needed, then, get the rotating angles in horizontal and vertical direction. The angle in horizontal direction of adjacent columns and the angle in vertical direction of adjacent rows should be equal. The calculating equation shows below:

$$
\begin{aligned}
V_{H} & =2 \cdot \arctan \left(\frac{\text { CmrWidth } \cdot \text { PxlSize }}{2 \cdot f}\right) \\
V_{V} & =2 \cdot \arctan \left(\frac{\text { CmrHeight } \cdot \text { PxlSize }}{2 \cdot f}\right) \\
C S & =\left\lceil\frac{\alpha}{\left(1-p_{x}\right) \cdot v_{H}}\right]+1 \\
r S & =\left\lceil\frac{\beta}{\left(1-p_{y}\right) \cdot v_{V}}\right\rceil+1 \\
\theta_{H} & =\frac{\alpha}{C S-1} \\
\theta_{V} & =\frac{\beta}{r S-1}
\end{aligned}
$$

where $V_{H}=$ the view angle of camera in horizontal direction $V_{V}=$ the view angle of camera in vertical direction $C m r W i d t h, C m r H e i g h t=$ the camera format with pixel as unit PxlSize $=$ the size of one pixel $\mathrm{cs}=$ the column number of image matrix $\mathrm{rs}=$ the row number of image matrix $\alpha=$ the rotating angle to cover with the block object in horizontal direction

$\beta=$ the rotating angle to cover with the block object in vertical direction $p_{x}=$ overlap in horizontal direction $p_{y}=$ overlap in vertical direction $\theta_{H}=$ the angle in horizontal direction of adjacent columns $\theta_{V}=$ the angle in vertical direction of adjacent rows \lceil\rceil$=$ round up

For the automatic triangulation, $p_{x}$ should be larger than $60 \%$, and $p_{y}$ be larger than $20 \%$. For reducing control points, it's better to set $p_{x}$ as $70 \%$ and $p_{y}$ as $30 \%$.

\subsection{Station setting strategy}

To realize the multi-view to solve the contradiction between matching and precision for the intersection angle, to organize the image data easily and to use fewer control points, we propose the design of classifying the stations into base stations and assistant stations. We need to divide the target into several blocks based on the width of the target, shoot distance, lens focus and the intersection angle needed. Then lay out stations with the principles that each block has two base stations and every two adjacent blocks share the same base station. In other words, the number of base stations is more than 1 to the block number. As the distance of base stations is large for large intersection angle, it influences the matching availability, we design assistance stations to reduce the baseline and transfer corresponding points. Not only the design implements multiview photography with high precision, but also adapts to measure objects with large width.

The number of blocks can be computed as: 
$B=D \cdot \tan \gamma$

$M=\operatorname{int}\left(\frac{S}{B}+0.5\right)+1$

Where $\mathrm{B}=$ the length of baseline

$\mathrm{D}=$ the shoot distance

$\gamma=$ the intersection angle of adjacent base stations

$\mathrm{S}=$ the width of the object

$\mathrm{M}=$ the number of blocks divided

And, the number of assistant stations between two adjacent base stations equals the integer of the setting intersection angle $\gamma$ of two adjacent base stations divided by the setting intersection angle $\gamma_{a d}$ of two adjacent assistant stations.

Because the intersection angle of adjacent base stations is the largest intersection angle in one block, which will decide the intersection precision. If the angle is too small, which leads to low precision, on the contrary, too large angle leads to low matching reliability as variation in image scale of stereo images. So $\gamma$ is no more than $45^{\circ}$. The intersection angle of two adjacent assistant stations affects the matching reliability, should be less than $15^{\circ}$. And when it's too small, the quantity of images increases sharply, which will need more time for data processing. Taking all into consideration, we advise $\gamma_{a d}$ to be $8^{\circ}$.

The principles of setting stations are that:

1) The first base station is right to the left edge of the target;

2) The shoot distance of every station is approximately equal;

3) The quantity of assistant stations between every two base stations is the same;

4) The distance of every two stations includes base stations and assistant stations should be equal.

Figure 2 shows the distribution of stations and the photograph range of each station. In figure2, the width of the target is $\mathrm{S}$ and the shoot distance is $\mathrm{D}$. The target is divided into 3 blocks as Block1, Block2, Block3. B1, B2, B3, B4 indicate the base stations. A11, A12 are assistant stations in block1, A21, A22 are assistant stations in block2, and A31, A32 are assistant stations in block3. Point a, b, c, d, e, f are points on the target. The lines show the photograph range of each station.

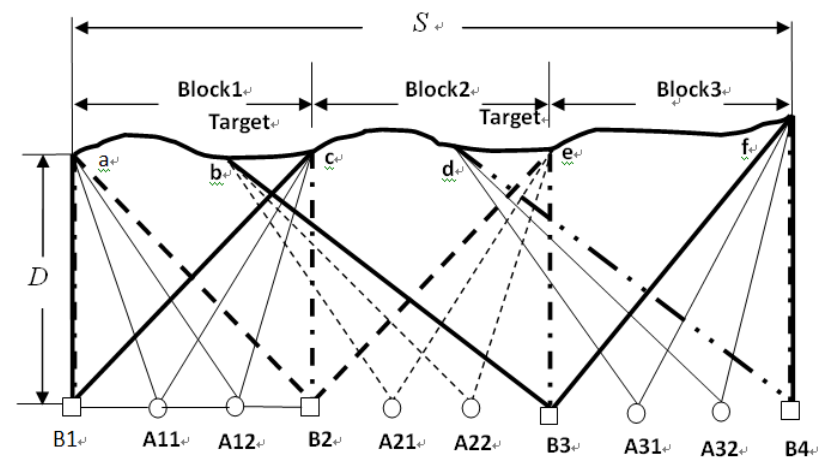

Figure2. The distribution of stations and the photograph range of each station.

When photographing, the first and last base stations need to photograph the first and last block, while other base stations as the shared station need to photograph two adjacent blocks. To assistant station, it only requires to photograph the corresponding block.
As the column and row number of each station's image matrix should be equal and the horizontal overlap of images gotten from base station is unchangeable, the range of image matrixes from stations in adjacent blocks is overlapping. That is to say the beginning point of scanning on stations excluded stations in the first block is the overlapping area. In figure2, the start point of scanning object for Station B1, A11, A12 is the point a, and the end point is point c. For Station B2, the scan range starts from point a and ends at point e. In block2, the range is from point $\mathrm{b}$ to point $\mathrm{e}$ for Station A21, A22. And for Station B3, the start and end point are point $\mathrm{b}$ and point $\mathrm{f}$ respectively. For Station $\mathrm{B} 4, \mathrm{~A} 31, \mathrm{~A} 32$, the range is from point $\mathrm{d}$ to point $\mathrm{f}$.

\subsection{The composition of the photograph scanner}

To ensure the efficiency and image quality, we study a scanner machine for data automatic acquisition specially. The scanner is consisted of main controller and rotating platform showed in figure 3. It can control the camera scanning photograph automatically after setting needed information, and record the camera posture information like the rotating angles in horizontal and vertical direction at the same time which can be used as auxiliary information to reduce the searching area in image matching.

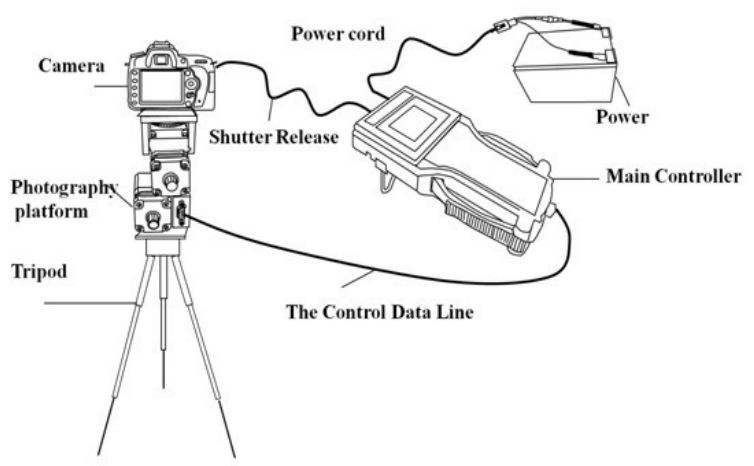

Figure3. the structure of photograph scanner

First, we fix the rotating platform on the triple, and put the camera on the platform. Then, connect the camera and platform to main controller, the main controller will control the camera to scanning photograph as design. Before photographing, we need to input the parameters required into the controller. The machine will calculate the row and column number as well as the rotating angle in horizontal and vertical direction and send the signal to rotate platform to control camera to rotate for image acquisition. The operator only need to move the machine to determined positions and make sure the range of the object to the station, the scanner will photograph and record information (such as the column and row number and the rotating angle) automatically in a log file.

\subsection{Data Procession}

After data acquisition, we need to transform the log file to the project file for data processing first. Then, compute points' space coordination automatically with classic aerial triangulation.

In scanning photograph, the images from different stations and having the same row and column rank construct strip, and can carry out stereoscopic analyses, for example relative orientation, model connection. So the sequence of image matching is image 
matching in strip, image matching between strips and image matching between blocks. The sequence of analyses is relative orientation and model connection in strips, strip mosaic, block mosaic, absolute orientation and self-calibration bundle adjustment.

The Scanning Photograph Data Processing Software developed by Wuhan University can realize the above aerial triangulation processing automatically, and it makes the data processed as a whole one with a small amount of control points in high efficiency and precision.

\section{EXPERIMENT}

Wuhan University cooperating with China Ordnance Industry Survey and Geotechnical Institute successfully applied the scanning photogrammetry system in the experiment for deformation monitoring of Wumen circumvallation in the Forbidden City. For confirming whether the circumvallation is deforming or not, the rivets with the length of $10 \mathrm{~cm}$ and $2 \mathrm{~cm}$ in diameter are hammered into wall distributed every $10 \mathrm{~m}$ in horizontal direction and every $5 \mathrm{~m}$ in vertical direction. The experiment is to get the rivets' coordination by scanning photogrammetry method and compare the result to them mapped by Total Station Topcon MS05A 0.5". And the accuracy of rivets' coordination mapped by Total Station is less than $\pm 3 \mathrm{~mm}$. We take the north wall and three sides of the flute showed in figure 5 for experiments with Canon EOS 5D Mark II digital camera whose picture format is $5616 \times 3744$ and pixel size is $6.4 \mu \mathrm{m}$.

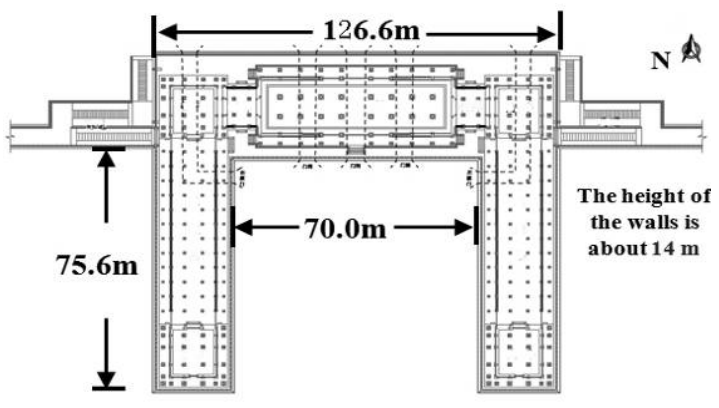

Figure5. The Architectural Drawing of the Wumen Circumvallation

We choose the north wall which is $126.6 \mathrm{~m}$ in width and 14 in height as the example to represent. As the height of the photograph scanner is much lower than the wall's, if we choose the long focus lens, the image will deform seriously for the large pitch. Generally, we selects $50 \mathrm{~mm}$ lens while the distance of station is $25 \mathrm{~m}$, so the ground resolution is $3.7 \mathrm{~mm}$. There are 15 orientation points and 51 check points. Figure6 shows control points' distribution. The orientation points are reflecting marks with side length of $30 \mathrm{~mm}$ and the interval is $30 \mathrm{~m}$ in horizontal and $5 \mathrm{~m}$ in vertical direction, whose side length showed on imageries are almost 15 pixels. For check points of rivets, the side length on imageries is nearly 10 pixels.

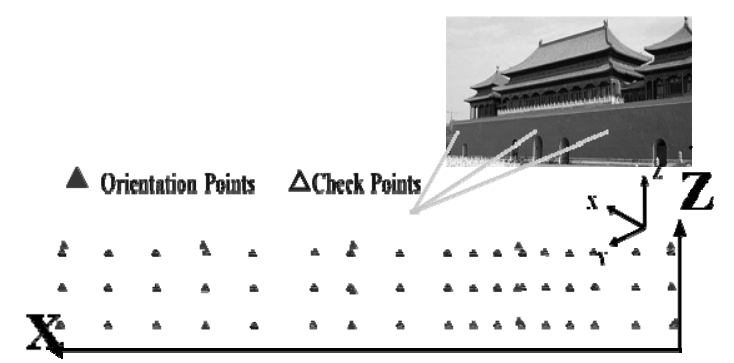

Figure6. The distribution of control points

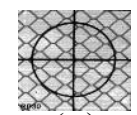

(a)

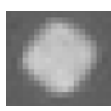

(b)

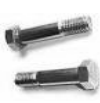

(c)

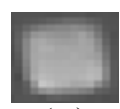

(d)
Figure7. Figure (a) is the reflecting mark's picture, and (b) is the imagery. Figure (c) is the picture of rivets, and (d) is the imagery.

We divide the target into 6 blocks, so there are 7 base stations. Between every two base stations, 3 assistant stations lay out. That is to say, it has 25 stations in total. For high precision goal, the overlaps in horizontal and vertical direction are set as $80 \%$ and $40 \%$ and the intersection angle between adjacent base stations is to be $40^{\circ}$. At last, 220 images are taken by the photo scanner. Table1 shows the accuracy. In this experiment the number of observations of a ground point can reach to 38 , and the largest intersection angle of feature points even to be $79.58^{\circ}$. It takes less than 120 minutes for data processing excluded the time of identifying control points' images coordination. The experiment's result meets the requirements of the monitoring in accuracy and efficiency. The experiment proves that, the scanning photogrammetry system can not only achieve automatic photograph with the scanner, but also improve mapping precision and reliability. What's more, the software can produce 3D point cloud of the target showed in figure 7 . Figure 8 is the Ortho-image of the north wall generated from $3 \mathrm{D}$ points.

\begin{tabular}{|c|c|c|}
\hline & Orientation points & $\begin{array}{c}\text { Check } \\
\text { points }\end{array}$ \\
\hline Number & 15 & 51 \\
\hline rmsX & 0.7 & 1.5 \\
\hline rmsY & 0.6 & 2.6 \\
\hline rmsZ & 1.0 & 3.4 \\
\hline Positional accuracy & 1.2 & 4.6 \\
\hline Relative Positional Accuracy & $1 / 21535$ & $1 / 6345$ \\
\hline
\end{tabular}

Table1. Precision of the north wall (unit: mm, sigma naught value: $1785 \mu \mathrm{m}$ )

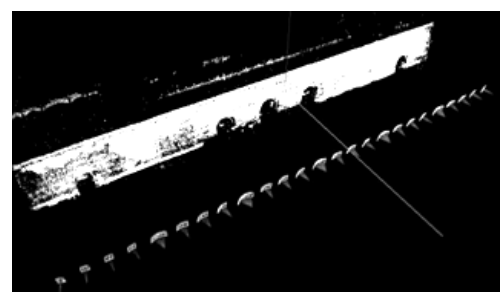

Figure7. 3D point cloud of the north wall 


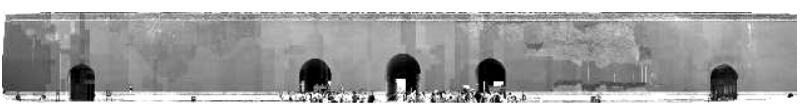

Figure8. Ortho-image of the north wall

The measurement of the rivets' coordination in the three sides of the flute imitated the process of the north wall. The shoot distance is also $25 \mathrm{~m}$. Table 2,3 and 4 show the results. But the results are not as good as in the experiment of the north wall. The main reason is that the color of the wall is similar to the rivet's as the paint on walls flaked, which mislead to identify check points' image coordination because the boundaries of rivets on images are blurry. In the future research, we will consider to use the camera with long focus lens and put the photograph scanner on a lift platform to make the rivets clear on images without serious deformation for a large pitch. It will improve the mapping precision because of exact check points' image coordination.

\begin{tabular}{|c|c|c|}
\hline & Orientation points & $\begin{array}{c}\text { Check } \\
\text { points }\end{array}$ \\
\hline Number & 13 & 20 \\
\hline $\mathrm{rmsX}$ & 0.4 & 8.8 \\
\hline $\mathrm{rmsY}$ & 0.7 & 4.7 \\
\hline $\mathrm{rmsZ}$ & 0.5 & 2.5 \\
\hline Positional accuracy & 1.0 & 10.3 \\
\hline Relative Positional Accuracy & $1 / 31254$ & $1 / 2801$ \\
\hline
\end{tabular}

Table2. Precision of the north wall of fluted circumvallation (unit: $\mathrm{mm}$, sigma naught value: $1.970 \mu \mathrm{m}$ )

\begin{tabular}{|c|c|c|}
\hline & Orientation points & $\begin{array}{c}\text { Check } \\
\text { points }\end{array}$ \\
\hline Number & 9 & 26 \\
\hline rmsX & 0.7 & 8.4 \\
\hline rmsY & 0.4 & 3.4 \\
\hline rmsZ & 1.9 & 2.5 \\
\hline Positional accuracy & 2.1 & 9.4 \\
\hline Relative Positional Accuracy & $1 / 15010$ & $1 / 3056$ \\
\hline
\end{tabular}

Table3. Precision of the west wall of fluted circumvallation (unit: $\mathrm{mm}$, sigma naught value: $1.946 \mu \mathrm{m}$ )

\begin{tabular}{|c|c|c|}
\hline & Orientation points & $\begin{array}{c}\text { Check } \\
\text { points }\end{array}$ \\
\hline Number & 9 & 24 \\
\hline rmsX & 1.5 & 4.5 \\
\hline rmsY & 0.9 & 3.1 \\
\hline rmsZ & 0.9 & 2.5 \\
\hline Positional accuracy & 2.0 & 6.0 \\
\hline Relative Positional Accuracy & $1 / 15165$ & $1 / 4729$ \\
\hline
\end{tabular}

Table4. Precision of the east wall of fluted circumvallation (unit: $\mathrm{mm}$, sigma naught value: $1.810 \mu \mathrm{m}$ )

\section{CONCLUSION}

In this paper, we present a new method of scanning photogrammetry which fixes the non-metric digital camera to rotate platform to control the camera rotating in both horizontal and vertical direction as photographing. It enlarges the view angle and intersection angle efficiently in the measurement of large targets, implements multi-view with the images organized easily by the method of classifying the stations into base stations and assistant stations, processes the data as the classic triangulation bundle adjustment procedure, and improves the reliability of image matching and redundant observations. The experiments prove that, the scanning photogrammetry system can not only achieve automatic photograph with the scanner, but also improve measurement precision and reliability. As a result, the new photogrammetry method can meet the needs of engineering survey such as the architecture measurement, deformation monitoring of cultural heritage and the tunnel measurement.

According to the complexity in close range and the process of scanning photogrammetry, in the future, we will take more effort to research the method of exactly identifying control points image coordination and the distribution of control points to gain high precision with fewer points.

\section{ACKNOWLEDGEMENTS}

The work described in this paper was supported by the National Science \& Technology Pillar Program with the project No. 2011BAH12B03.

\section{References}

Chandler, J., 1999. Effective application of automated digital photogrammetry for geomorphological research. EARTH SURFACE PROCESSES AND LANDFORMS, 24(1), pp.51-63.

Feng, W., 1982. The application of non-metric camera in closerange photogrammetry. Railway Investigation and Surveying, (0 $4)$.

Fraser, C.S., Shortis, M.R. and Ganci, G., 1995. Multi-sensor system self-calibration, In: Videometrics IV. SPIE Proceedins.

Jiang, R.N. and Jauregui, D.V., 2010. Development of a digital close-range photogrammetric bridge deflection measurement system. Measurement, 43(10), pp.1431-1438.

Ke, T., Zhang, Z.X. and Zhang, J.Q., 2007. Panning and multibaseline digital close-range photogrammetry. In: MIPPR2007,November 2007, Wuhan China.

Ke, T., 2008. Panning and Multi-baseline Digital Close-range Photogrammetry. Wuhan Unviersity, Wuhan, China.

Luhmann, T., 2010. Close range photogrammetry for industrial applications. ISPRS Journey of Photogrammetry and Remote Sensing, 65(6), pp.558-569.

Peipe, J. and Schneider, C.T., 1995. High-resolution still video camera for industrial photogrammetry. Photogrammetric Record, 15(85), pp. 135-139.

Zhang Z.X., 2007. From Digital Photogrammetry Workstation (DPW) to Digital Photogrammetry Grid (DPGrid). Geomatics and Information Science of Wuhan University,(7), pp. 565-571.

Zhang Z.X., Yang C.S, Zhang J.Q., et al. 2007. Multi-Baseline Digital Close-Range Photogrammetry. Geospatial information, 5(1), pp.1-4. 